\title{
Dissociated Signals in Human Dentate Gyrus and CA3 Predict Different Facets of Recognition Memory
}

\author{
Zachariah M. Reagh, ${ }^{1,2,3}$ Joseph Watabe, ${ }^{4}$ Maria Ly, ${ }^{4}$ Elizabeth Murray, ${ }^{1,2,3}$ and $\oplus^{\circ}$ Michael A. Yassa ${ }^{1,2,3}$ \\ ${ }^{1}$ Department of Neurobiology and Behavior, ${ }^{2}$ Institute for Memory Impairments and Neurological Disorders, and ${ }^{3}$ Center for Neurobiology of Learning and \\ Memory, University of California, Irvine, Irvine, California 92697, and ${ }^{4}$ Department of Psychiatry, University of Iowa, Iowa City, Iowa 52242
}

\begin{abstract}
A wealth of evidence has implicated the hippocampus and surrounding medial temporal lobe cortices in support of recognition memory. However, the roles of the various subfields of the hippocampus are poorly understood. In this study, we concurrently varied stimulus familiarization and repetition to engage different facets of recognition memory. Using high-resolution fMRI (1.5 mm isotropic), we observed distinct familiarity and repetition-related recognition signal profiles in the dentate gyrus (DG)/CA3 subfield in human subjects. The DG/CA3 demonstrated robust response suppression with repetition and familiarity-related facilitation. Both of these discrete responses were predictive of different aspects of behavioral performance. Consistent with previous work, we observed novelty responses in CA1 consistent with "match/mismatch detection," as well as mixed recognition signaling distributed across medial temporal lobe cortices. Additional analyses indicated that the repetition and familiarity-related signals in the DG/CA3 were strikingly dissociated along the hippocampal longitudinal axis and that activity in the posterior hippocampus was strongly correlated with the retrosplenial cortex. These data provide novel insight into the roles of hippocampal subfields in support of recognition memory and further provide evidence of a functional heterogeneity in the human DG/CA3, particularly along the longitudinal axis.
\end{abstract}

Key words: CA3; dentate; fMRI; hippocampus; memory; recognition

\section{Introduction}

Recognition memory refers to the ability to judge the previous occurrence of stimuli (Brown and Aggleton, 2001) and is a critical mechanism for learning from one's experiences and adapting to one's environment. This function has been primarily attributed to structures in the medial temporal lobe (MTL), consisting of the hippocampus and adjacent entorhinal (EC), perirhinal (PRC), and parahippocampal (PHC) cortices (Eichenbaum et al., 2007). However, the exact computational and functional nature of these regions is still a matter of ongoing research.

Brown and Aggleton (2001) previously characterized several recognition-related changes in neural firing based on neurophysiological recordings in nonhuman primates. These include familiarity (suppressed activity to familiar stimuli only, regardless of repetition), recency (suppressed activity to repetition, regardless of familiarity), and novelty (suppressed activity to repetition of novel stimuli and decreased firing rate to familiar stimuli). These signals have been observed in various regions along the parahippocampal gyrus (Buffalo et al., 1998; Murray and Bussey, 1999;

Received July 8, 2014; revised Aug. 14, 2014; accepted Aug. 21, 2014.

Author contributions: M.A.Y. designed research; J.W., M.L., and E.M. performed research; Z.M.R. analyzed data; Z.M.R. and M.A.Y. wrote the paper.

This work was supported by National Institute on Aging Grants P50 AG05146 and R01 AG034613. Z.M.R. is supported by National Science Foundation Graduate Research Fellowship DGE-1232825. We thank Fellows of the Center for Neurobiology of Learning and Memory for helpful discussions regarding the data. We especially thank Craig Stark and Shauna Stark for their input.

Correspondence should be addressed to Dr. Michael A. Yassa, Department of Neurobiology and Behavior, University of California, Irvine, 213 Qureshey Research Laboratory, Irvine, CA 92697-3800. E-mail: myassa@uci.edu.

DOI:10.1523/JNEUROSCI.2779-14.2014

Copyright $\odot 2014$ the authors $\quad 0270-6474 / 14 / 3413301-13 \$ 15.00 / 0$
Wan et al., 1999), and a single region may exhibit many different firing patterns in support of recognition without clear spatial topography (Brown and Aggleton, 2001).

Compared with cortical regions, recognition signals in the hippocampus are less well understood. Some previous studies of the hippocampus have revealed response modulation associated with recognition (Wan et al., 1999; Jutras and Buffalo, 2010), and hippocampal lesions have been found to produce recognition memory deficits (Clark et al., 2000; Manns et al., 2003). However, this is a complex and unresolved issue given that hippocampal lesions have also been observed to have selective effects on "recollection" (context-rich retrieval of an event in memory) but not "familiarity-based" memory judgments (a general, decontextualized sense of remembering; Düzel et al., 2001; Yonelinas et al., 2002; Fortin et al., 2004). As such, the hippocampus likely contributes to, but is not equally necessary for, all forms of recognition memory (Barker and Warburton, 2011).

A common result in human neuroimaging studies is a form of novelty signal profile in the hippocampus, often referred to as "match/mismatch" signaling. Here, responses are modulated by a stimulus having been previously presented and "matching" or "mismatching" with respect to future presentations (Kumaran and Maguire, 2006, 2007; Duncan et al., 2009), particularly in subregion CA1 (Duncan et al., 2012). Another often-studied phenomenon is suppression of BOLD activity in the hippocampal dentate gyrus (DG) and CA3 subregions with stimulus repetition (Bakker et al., 2008; Lacy et al., 2011). This may be a type of recognition signal such that the lack of a novelty effect signifies a lesser need to encode a stimulus. Whereas DG/CA3 activity is reduced in response to repeated items, it fails to do so in response 


\section{a}

Scenes prefamiliarized 24 hours prior to scanned recognition test
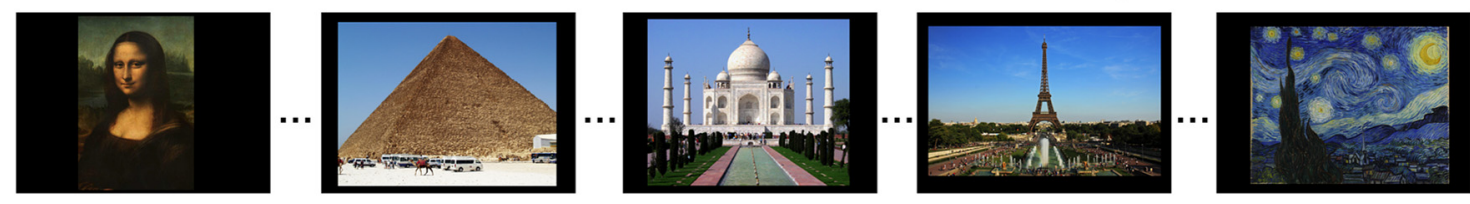

b Novel Foil
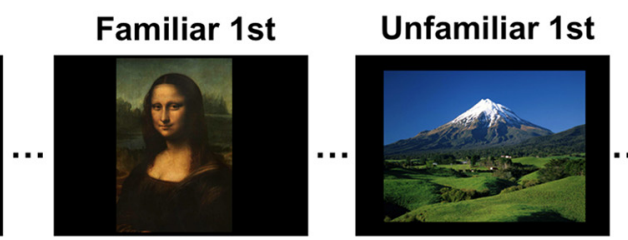

Familiar 2nd

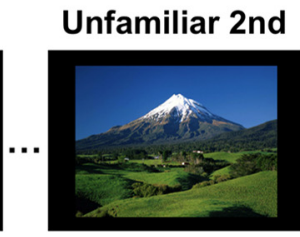

C

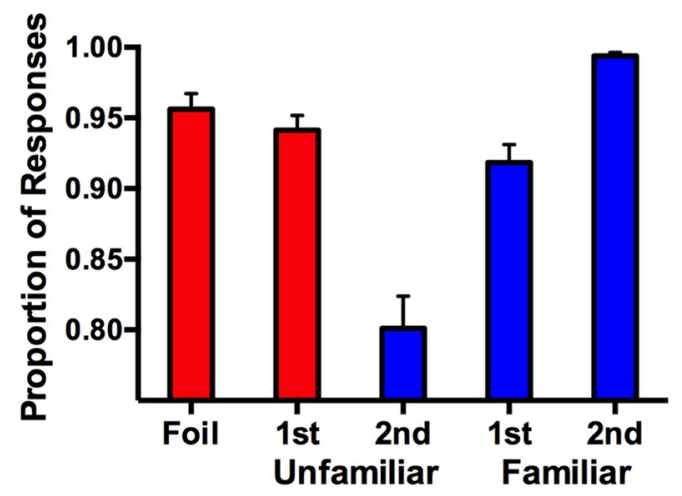

d

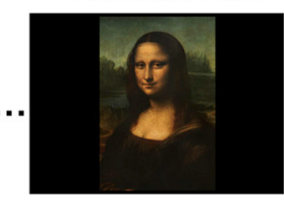

Correct Rejections

Target Hits

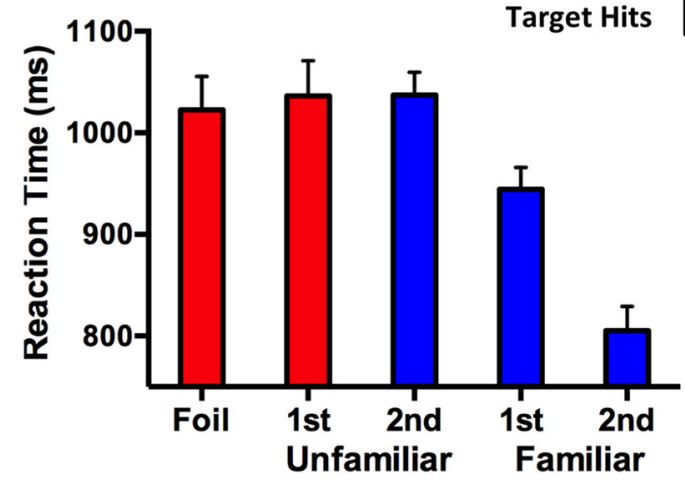

Figure 1. Task design and behavioral results. $\boldsymbol{a}$, Study phase in which participants were familiarized with 80 widely recognizable scenes $24 \mathrm{~h}$ before the test. $\boldsymbol{b}$, Test phase in which participants were tested via "Yes/No" recognition. Trials included Familiar first (Familiar first) and second (Familiar second) presentations and Unfamiliar first (Unfamiliar first) and second (Unfamiliar second) presentations ( 80 trials each), as well as Novel Foils ( 160 trials). Proportion of responses (c) and reaction time (d) indicate an overall benefit of familiarization for hit rate, which is further aided by repetition. Correct rejection of Foils and Unfamiliar first stimuli did not differ.

to novel information, even if highly similar to information in memory.

Much of our knowledge about the importance of these structures is based on studying the effects of lesions to MTL structures (Zola-Morgan et al., 1989; Suzuki et al., 1993; Murray et al., 1998; Norman and Eacott, 2005), and recorded activity (e.g., via fMRI) has been interpreted as confirming their respective roles (Yonelinas et al., 2005; Yassa and Stark, 2008; Johnson et al., 2009; Diana et al., 2012). However, there is little existing evidence of the behavioral relevance of particular signals measured in the hippocampus (for one example, see Hannula and Ranganath, 2008). In the present study, we sought to observe and characterize recognition signals in human hippocampal subfields and MTL cortices, as well as the behavioral relevance of these signals.

\section{Materials and Methods}

Participant selection. Twenty-three healthy volunteers were recruited from the local student community by institutional E-mail and flyer advertising. All participants were compensated for their time, and informed consent was obtained in accordance with the local Institutional Review Board. Exclusion criteria included any major medical conditions (e.g., heart disease, diabetes), neurologically active medications, and current diagnosis of a mental or psychiatric disorder (e.g., schizophrenia, depression). Of the original sample, four participants were excluded because of technical issues, and one was excluded because of excessive motion in the scanner. This resulted in a final sample of 18 healthy participants $(8$ females, 10 males; mean \pm SD age, $23.7 \pm 3.9$ years).

Scene repetition task. Two types of recognition are particularly relevant to this investigation and can be used to dissociate different signal profiles. One is recognizing stimuli that are familiarized (preexperimentally), and the other is recognizing stimuli that are repeated within the experiment. As such, participants were tested on unfamiliar stimuli that were repeated within session and on stimuli that were preexperimentally familiarized and repeated.

The behavioral task consisted of two phases. The first phase was an out-of-scanner training phase in which participants were familiarized with 80 pictures of widely recognizable scenes (e.g., the Mona Lisa; Fig. $1 a)$. These recognizable stimuli were chosen to facilitate recognition and maximize familiarization with the images to be tested. Participants were instructed to indicate via button press whether they did or did not recognize the scene depicted and to study the image. Subjects had $10 \mathrm{~s}$ to view each image and read the associated information (a verbal label for the item), with $1 \mathrm{~s}$ between each image.

Approximately $24 \mathrm{~h}$ later, subjects completed an in-scanner test phase (Fig. 1b). In this phase, participants viewed 80 familiarized scenes for the first time within session (Familiar first) and a second time (Familiar second). Participants also viewed 80 unfamiliar scenes for the first time (Unfamiliar first) and a second time (Unfamiliar second). Finally, participants viewed 160 novel foil images that were never repeated in the experiment (Foils). In total, this phase consisted of 400 trials. Scenes were viewed for $2500 \mathrm{~ms}$ each, with an interstimulus interval of $500 \mathrm{~ms}$. During the presentation of each scene, subjects were tasked with judging whether that scene was encountered before or not (including those recognized from the study session on the previous day and those repeated within the test session).

In the context of this experiment, we have operationalized "familiar" stimuli such that participants were trained on these stimuli before the test session. We note that, importantly, this is distinct from dual process models of memory, which describe remembering in terms a general sense of familiarity versus an episodic "recollective" experience (Yonelinas et al., 2005; Daselaar et al., 2006; Diana et al., 2007; Johnson et al., 2009; 
Ranganath and Ritchey, 2012). As such, our operational use of "familiar" applies selectively to stimuli that were trained. This designation further applies because some iteration of these stimuli have probably been seen on numerous past occasions (this potentially involves a greater semantic component to these stimuli as well, but nonetheless enhances familiarization in line with our manipulation). We also note that, with respect to our experimental design, Unfamiliar first scenes were no different from Foils, although these were treated as different trial types in analysis for baseline and quality-assurance purposes (see below, Image processing and analysis). The in-scanner test phase took $20 \mathrm{~min}$ and was divided into four runs of $5 \mathrm{~min}$ each. Trials were presented in random order, and each run contained all four conditions. We used PsychToolbox (Brainard, 1997) via MATLAB (MathWorks) for stimulus presentation and behavioral response collection.

Analysis of behavioral data. In analyzing the proportion of correct responses, the data were divided into correct rejection of previously unseen images (novel Foils and Unfamiliar first correct rejections) and target hits on previously seen images (Familiar first hits, Familiar second hits, and Unfamiliar second hits). Given the different nature of these types of correct responses, we chose to make comparisons within response type rather than across responses. We additionally collected response latency and analyzed this variable using the same procedures as accuracy (correct rejections and target hits separately).

MRI data acquisition. fMRI data were collected using a 3 tesla Philips scanner equipped with a 32-channel SENSE head coil using both higherorder shims and SENSE parallel acceleration techniques. Functional images were collected using a high-speed echo-planar single-shot pulse sequence with a field of view of $96 \times 96 \mathrm{~mm}$, flip angle of $70^{\circ}$, SENSE factor of 2, TR of $1500 \mathrm{~ms}$, TE of $35 \mathrm{~ms}$, a 4 TR initial skip, and resolution of $1.5 \mathrm{~mm}$ isotropic. In each run, 19 triple-oblique axial slices were acquired aligned to the principle axis of the hippocampus bilaterally. This resulted in partial acquisitions of the brain but fully included the hippocampus and surrounding MTL cortices, as well as partial coverage of cingulate and parietal cortices, including the retrosplenial cortex (RSC).

We additionally collected a high-resolution structural MPRAGE scan with a field of view of $240 \times 240 \mathrm{~mm}$, flip angle of $9^{\circ}$, TR of $12 \mathrm{~s}$, TE of 5.9 s, matrix size of $384 \times 384$, and $0.65 \mathrm{~mm}$ isotropic resolution. SENSE parallel imaging was used in two directions $(2 \times 1.5)$, yielding an overall volume of 231 slices.

Image processing and analysis. Functional image preprocessing and univariate analyses were performed with AFNI (Analysis of Functional NeuroImages) software (Cox, 1996). Significant motion events (3 mm rotation or $2 \mathrm{~mm}$ translation or greater in any direction) and the preceding and following TRs were censored and excluded from analyses. Functional images were corrected for slice timing and head motion and were detrended across acquisitions using a first-order (linear) polynomial to further reduce the influence of drift in the scanner signal over time. Volumes were aligned to Talairach space, and partial functional acquisitions were aligned to structural volumes and were smoothed with a $2 \mathrm{~mm}$ Gaussian kernel to improve statistical fit.

Cross-participant alignment was performed using ANTS (Advanced Normalization Techniques) software (Avants et al., 2009). Before group registration, each participant's structural and functional data were aligned to Talairach space. The structural and functional volumes were then aligned to a common template $(0.65 \mathrm{~mm}$ isotropic). Regions of interest (ROIs) were defined anatomically in accordance with the atlas of Duvernoy (1998). Cortical ROIs included the PRC, PHC, EC, and RSC. Hippocampal ROIs included CA1, subiculum, and a combined DG/CA3 subregion. There are several anatomically and functionally informed reasons underlying our decision to avoid attempts at separately analyzing the DG and CA3 in our data. First, a fairly sizeable portion of CA3 is in fact encapsulated within the DG in the coronal plane, which is extremely difficult (if not altogether impossible) to visually distinguish even with $0.55 \mathrm{~mm}$ isotropic structural MRI. Therefore, at $1.5 \mathrm{~mm}$ BOLD resolution and given the inherent spatial blur of fMRI data, the validity of separating DG from CA3 signals would be tenuous at best. Second, the anatomy of the two regions is somewhat atypical in that DG granule cells, although numerous, fire sparsely, and CA3 pyramidal cells are connected recurrently in a positive feedback network, with both regions under tight inhibitory control (Yassa and Stark, 2011). Furthermore, based on extensive work in rodents, we know that activity in CA3 is heavily influenced by activity upstream in the DG. These issues are regularly and actively discussed at the Hippocampal Subfield Segmentation Summit meetings (HS3 Conference; http://hippocampalsubfields.com), an international collaborative effort dedicated to deriving an anatomically validated, unified protocol for hippocampal segmentation on MRI scans.

3D deconvolution (Cox, 1996) based on multiple linear regression was performed with respect to trial type (i.e., familiarization and repetition). Novel images that were never repeated (i.e., Foils) were not modeled and were thus used as the implicit baseline condition. Importantly, these trials do not in principle differ from unfamiliar first presentation (Unfamiliar first correct rejections) trials such that these trials should not differ from baseline (an internal control condition that bolsters the validity of differences from baseline observed in other modeled trial conditions). Parameter estimates ( $\beta$ coefficients) resulting from the regression estimated activity versus baseline and the sum of $\beta$ coefficients over the expected hemodynamic response (3-12 s after trial onset) were taken as the model's estimate of the relative response to each trial type. Signal was averaged across all voxels within each anatomical ROI. The data were subjected to a series of planned $2 \times 2$ repeated-measures ANOVAs with familiarization (familiar vs unfamiliar) and repetition (first vs second presentation) as fixed factors. Because voxels were selected on a purely anatomical basis, voxel selection biases are avoided entirely.

Beyond a general assessment of signal profiles across regions, we sought to determine the extent to which each region featured the three targeted recognition signal profiles. Toward this end, we conducted a second-level $2 \times 2$ ANOVA with the same parameters as the analysis described above. We thresholded the resulting statistical map using a voxelwise error rate of $p<0.05$ based on the omnibus $F$. This served to exclude voxels from the analyses whose activity was probabilistically attributable to noise (i.e., null voxels). In the remaining voxels, we ran a priori specified contrasts to assess the extent to which active voxels conformed significantly to particular canonical recognition signal profiles. We define contrast weights $(\mathrm{cw})$ for these directed hypothesis tests below. First, we created a contrast mask for familiarization [Unfamiliar first correct rejections (cw:1) and Unfamiliar second hits (cw:1) > Familiar first hits (cw: -1$)$ and Familiar second hits $(\mathrm{cw}:-1)$ ], recency [Familiar first hits (cw:1) and Unfamiliar first correct rejections (cw:1) > Familiar second hits (cw:-1) and Familiar second hits (cw:-1)], and novelty [Unfamiliar first correct rejections (cw:3) > Unfamiliar second hits (cw: -1$)$, Familiar first hits (cw:-1), and Familiar second hits (cw:-1)] across all ROIs. Despite the fact that our contrasts were a priori planned contrasts, we conservatively opted to use a multiple comparison correction; thus, all results were Bonferroni-corrected at a final critical $\alpha$ of $p=0.017$ (accounting for three mask conditions). Each voxel was designated to whichever contrast most robustly captured its variance (i.e., the largest $F$ statistic in a winner-take-all manner), and if none of the three specific contrasts were significantly evident, we designated that voxel into an other/none category. This resulted in an approximate percentage of voxels conforming mostly to any of the three contrasts, or other/none. We note that, under this thresholded approach, a healthy proportion of other/none voxels are anticipated. This could be attributable to signal profiles we did not anticipate and model or simply noisy voxels that we failed to filter out with our masks. We additionally note that the approach taken in this analysis is mostly distinct from our first-level analysis and, although potentially redundant in overall results, does not affect the type 1 error rate of the first-level ANOVA for a given ROI. Although we indeed took note of the extent to which contrast masks shared overlapping voxels, the only ROI in which this overlap exceeded $25 \%$ of the surviving voxels was the PHC, but this did not affect the conclusions drawn about this or any other ROI (see Results).

Statistical testing. We compared accuracy and response latency of correct rejections using an independent samples $t$ test for each measure (because these measures comprise separate families of data, no corrections are necessary). We note that these comparisons are expressly expected not to differ given that, during the initial presentation of an unfamiliar item, it is essentially no different from a novel Foil trial. Target hits and respective response latencies were each assessed with a repeated- 
measures ANOVA with trial type as the single within-subjects factor. Significance was assessed with correction for nonsphericity of error. A significant omnibus $F$ statistic was interrogated post hoc using Tukey's HSD, which accounts for $\alpha$ inflation given multiple pairwise comparisons. All tests were two tailed, with an initial $\alpha$ of $p=0.05$ (before any adjustments).

Statistical testing of $\mathrm{fMRI}$ data was first evaluated via a three-way ANOVA $(2 \times 3 \times 4$ for hemisphere $\times$ region $\times$ trial type $)$ across hippocampal subfields and across MTL cortices to observe general effects and interactions across the hippocampal and cortical datasets. Although informative, this yields a highly complex view of the dataset. Specific and more tractable analyses were performed using a repeated-measures ANOVA for average $\beta$ coefficients over each ROI as a function of trial condition. Post hoc comparisons after a significant ANOVA were conducted using contrasts (chosen over Tukey's test given four levels of the independent variable). Contrasts weigh the means of the respective conditions to generate a sum of squares for each comparison, which is divided by the appropriate error term from the omnibus ANOVA to generate an F statistic. Analyses were Greenhouse-Geisser corrected for nonsphericity when appropriate. Post hoc contrasts were corrected for $\alpha$ inflation using Scheffé's method, which adjusts the critical $F$ statistic on the basis of the number of conditions modeled in the initial ANOVA (for the present analyses, $N-1$ conditions $=3$; critical $F=4.451$; thus, critical $\left.F_{S}=13.353\right)$. All correlational analyses were Bonferroni corrected for a given family of data, and tests reported as being significant surpassed the adjusted threshold. As with behavioral analyses, all tests were two tailed, with an initial $\alpha$ of $p=0.05$ (before any adjustments). Effect sizes are reported as partial $\eta$-squared values $\left(\eta_{p}^{2}\right)$.

ANOVAs over behavioral variables and mean ROI activity were conducted in SPSS version 22.0 (IBM SPSS). Post hoc analyses were performed and adjusted for multiple comparisons and nonsphericity using in-house spreadsheets created in Microsoft Excel.

\section{Results \\ Behavior}

Accuracy

An independent samples $t$ test revealed that subjects did not differ in the proportion of novel Foil correct rejections and Unfamiliar first correct rejections $\left(t_{(17)}=1.01, p=0.331\right.$; Fig. $\left.1 c\right)$. Although the unfamiliar trials are repeated at a later time, Unfamiliar first trials are essentially novel Foils in their first iteration. As such, this result is expected and is essentially confirmatory.

A one-way repeated-measures ANOVA revealed a significant effect of trial type on hit rate $\left(F_{(2,34)}=68.609, p<0.001\right.$; Fig. $\left.1 c\right)$. Post hoc contrasts via Tukey's HSD (critical $q=3.11$ ) revealed that subjects demonstrated a greater proportion of Familiar second hits than Familiar first hits $(q=9.43, p<0.05)$, as well as Unfamiliar second hits $(q=17.34, p<0.05)$. Furthermore, hit rate was greater for Familiar first items than Unfamiliar second items $(q=9.28, p<0.05)$. These results suggest that pretest familiarization conferred a general benefit for recognition and that a second repetition of a familiarized item conferred additional benefit, boosting performance to ceiling for Familiar second trials.

\section{Reaction times}

An independent samples $t$ test revealed that subjects' mean response latency did not differ between novel Foil correct rejections and Unfamiliar first correct rejections $\left(t_{(17)}=0.77, p=0.292\right.$; Fig. 1d). As per the analogous test on correct rejection rates above, this result confirms that subjects did not differ in reaction times across these trials.

A second ANOVA (sharing the same design) revealed a significant effect of trial type on response latency for target hits $\left(F_{(2,34)}\right.$ $=25.84, p<0.001$; Fig. $1 d)$. Post hoc contrasts via Tukey's HSD (critical $q=3.11$ ) revealed that subjects were significantly faster at Familiar second hits than Familiar first hits $(q=7.26, p<$ $0.05)$, as well as Unfamiliar second hits $(q=13.27, p<0.05)$. Familiar first hit trials were furthermore significantly faster than Unfamiliar second hit trials $(q=3.95, p<0.05)$. In line with the previous analysis on hit rate, this shows that, in addition to increased target recognition, familiarization was associated with faster response times and that a second repetition of a familiarized item elicited notably faster accurate responses than other trial types.

\section{fMRI}

Activity did not differ in any ROI between Unfamiliar first correct rejections trials and baseline (Foils; $t$ test against zero, all $p>$ $0.05)$. Consistent with the absence of behavioral differences, this supports our use of novel trials as a baseline condition against which memory-related signals are measured and suggests that this baseline was validly established. We did not observe significant modulations in either left or right subiculum (omnibus ANOVA, both $p>0.05$ ), and that data will thus not be discussed. However, data across hemispheres for this ROI can be viewed in Figure $2, e$ and $h$.

We first took a very general approach to the data and performed a three-way ANOVA $(2 \times 3 \times 4$ for hemisphere $\times$ region $\times$ trial type) across hippocampal subfields (regions: CA1, subiculum, and DG/CA3) and MTL cortices (regions: PRC, PHC, and EC) to assess significant effects across the dataset. Segmentation of hippocampal subfields and MTL cortices can be seen in representative anterior (Fig. $2 a$ ) and posterior (Fig. $2 b$ ) slices.

Across hippocampal subfields, we observed a significant effect of region $\left(F_{(2,30)}=6.268, p=0.01\right.$ corrected $)$ and trial type $\left(F_{(3,45)}=4.940, p=0.006\right.$ corrected $)$ but no effect of hemisphere $\left(F_{(1,15)}=0.742, p=0.403\right.$ corrected $)$. We further observed a significant interaction between region and trial type $\left(F_{(6,90)}=\right.$ 5.887, $p<0.001$ corrected). No other interactions reached significance. Thus, in general, hippocampal subfields were indeed modulated by at least some aspect of recognition memory in this task, and this extent of this modulation varied across our ROIs.

The ANOVA in MTL cortices revealed significant effects of region $\left(F_{(2,30)}=5.044, p=0.016\right.$ corrected $)$ and trial type $\left(F_{(3,45)}\right.$ $=9.702, p=0.001$ corrected) and a modest effect of hemisphere $\left(F_{(1,15)}=4.775, p=0.045\right.$ corrected $)$. Similarly to hippocampal subfields, we observed an interaction between region and trial type $\left(F_{(6,90)}=5.497, p<0.001\right.$ corrected $)$. We additionally found a significant interaction between region and hemisphere in MTL cortices, as may be expected given an effect of hemisphere $\left(F_{(2,30)}\right.$ $=5.202, p=0.012$ corrected). Thus, MTL cortices appear to have been modulated by the recognition memory task as well, with potential laterality of effects.

The above analyses indicate two important points. First, activity in our hippocampal and cortical ROIs were significantly modulated by the recognition task conditions. Second, these modulations significantly varied across the ROIs. We next sought to parse these modulations as a function of trial type, and assess responses to familiarized stimuli and repetition. Toward this end, we interrogated signal profiles across each ROI individually. For regions in which activity was highly consistent across hemispheres, data were collapsed for the sake of more straightforward analyses (but Figs. 2 and 7 display data from each hemisphere for hippocampal ROIs and MTL cortical ROIs, respectively). For regions with differences in responses across trial types, left and right hemispheric data were considered separately to assess their unique signal profiles. 

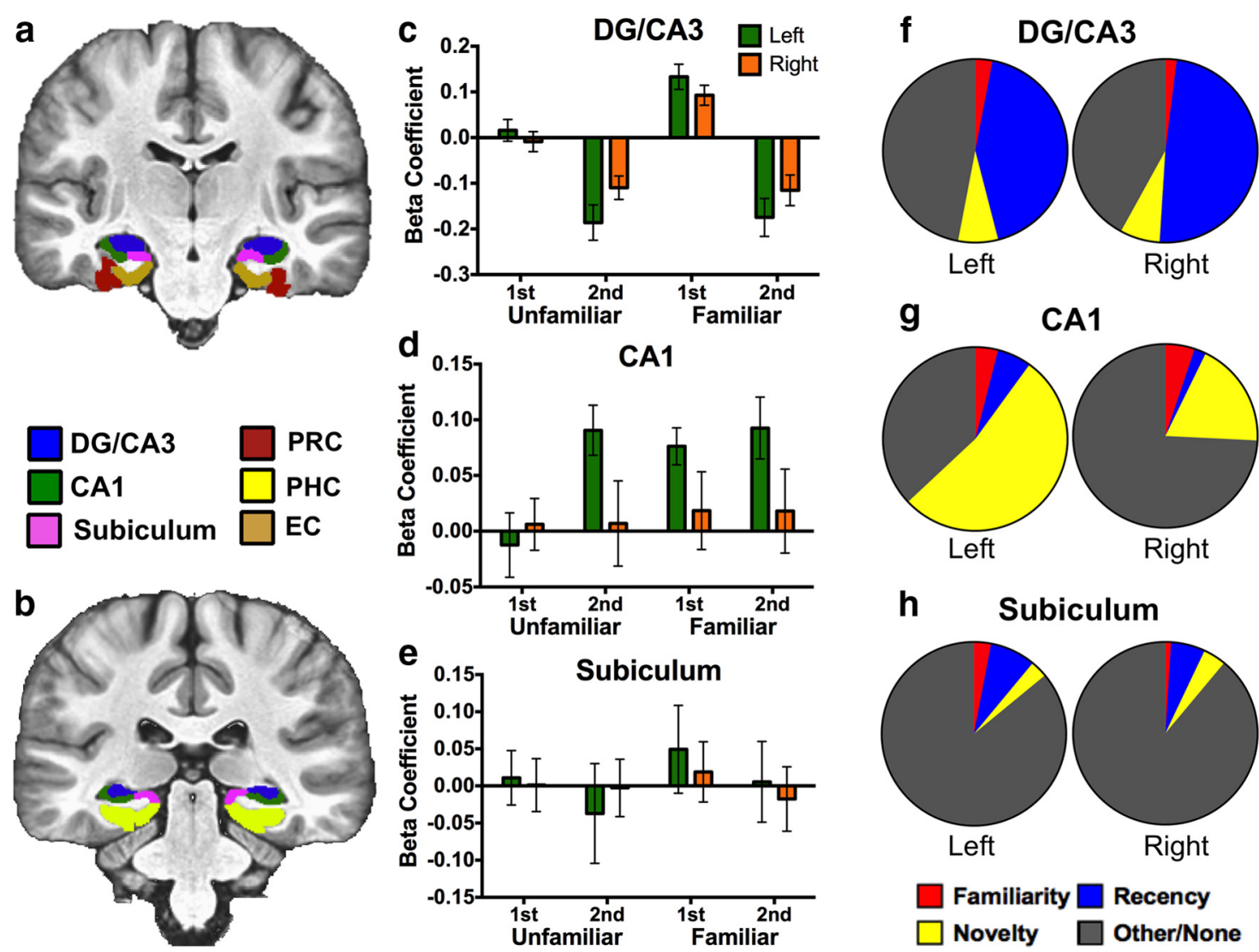

h Subiculum

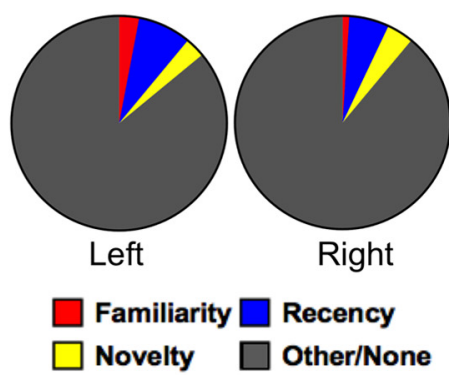

Figure 2. Recognition signals in hippocampal subfields. Segmentation is displayed in two representative anterior (a) and posterior ( $\boldsymbol{b})$ slices. Activity during Novel Foil trials was used as baseline (zero). c, Activity in the bilateral DG/CA3 was highly consistent with recency signaling with the exception of above-baseline activity during Familiar first trials, which are parsed in a later analysis. $\boldsymbol{d}$, Activity in the left CA1 was consistent with match/mismatch signaling, whereas the right CA1 showed no significant recognition signal profiles. $\boldsymbol{e}$, Activity in the bilateral subiculum showed no significant differences from baseline. $\boldsymbol{f}$ - $\boldsymbol{h}$, Percentage of voxels conforming to each of the three canonical recognition signal profiles or other/none across hippocampal subfields at a familywise threshold of $p=0.05$. Voxels were designated based on whichever contrast yielded the largest significant $F$ statistic.

\section{Hippocampal ROIs}

The left CA1 demonstrates novelty signaling

We did not observe significant deviations from baseline in the right CA1 $\left(F_{(1,17)}=0.172, p>0.05\right)$. Laterality of effects in BOLD fMRI data is not uncommon and varies from study to study. It is often difficult to draw conclusions about hemispheric effects, which can be a product of significance thresholding or failure of an ROI in a particular hemisphere to sufficiently demonstrate an effect via the BOLD signal. That is, it is possible that memory-related processing is occurring at the neuronal level and simply is not being reflected in the BOLD signal. With this in mind, additional analyses in CA1 are limited to the left hemisphere. A repeated-measures ANOVA in the left CA1 revealed significant effects of familiarization $\left(F_{(1,17)}=\right.$ 4.519, $\left.p=0.048, \eta_{p}^{2}=0.21\right)$ and repetition $\left(F_{(1,17)}=10.654, p=\right.$ $\left.0.005, \eta_{p}^{2}=0.385\right)$. An interaction was marginal but not significant $\left(F_{(1,17)}=3.948, p=0.063, \eta_{p}^{2}=0.188\right)$ (Fig. $\left.2 d\right)$. Post hoc contrasts revealed that the left CA1 robustly demonstrated novelty signaling $\left(F_{(1,17)}=15.453, p<0.05\right.$ corrected $)$. We note that modulations here were globally above baseline, although we cannot draw strong conclusions based on the directionality of the BOLD signal. Interestingly, despite significant main effects, specific contrasts assessing familiarity and recency signals were not significant $(p>0.05$ corrected).

We next quantified voxels as a function of signal profile. This analysis revealed that a majority (53\%) of voxels in the left CA1 indeed conformed to novelty signaling (Fig. $2 g$ ). Comparatively few voxels conformed to the familiarity or recency signal contrasts. The observation of novelty signaling in CA1 is consistent with previous reports of match/mismatch detection in this hippocampal subfield (Duncan et al., 2012). However, this terminology carries computational connotations beyond a novelty signal that we cannot confidently speak to in our data. Additionally, our experimental design and novelty response profile have several important distinctions. We note that our familiarized stimuli are exact repetitions and our novel stimuli are (to our knowledge) entirely novel. As a consequence, our global novelty-related modulation may demonstrate properties of either match or mismatch signals but not capture a binary match/ mismatch computation per se (i.e., there is little if any partial overlap among stimuli on which to perform such a computation).

The DG/CA3 shows heterogeneous recognition signals

Activity in the DG/CA3 was highly consistent across the left and right hemispheres and was thus collapsed for the following analyses (for visualization of both hemispheres, see Fig. 2c). A repeated-measures ANOVA revealed significant effects of familiarization $\left(F_{(1,17)}=12.533, p=0.003, \eta_{p}^{2}=0.424\right)$ and repetition $\left(F_{(1,17)}=34.861, p<0.001, \eta_{p}^{2}=0.672\right)$, as well as a significant interaction $\left(F_{(1,17)}=8.513, p=0.01, \eta_{p}^{2}=0.334\right.$; Fig. $2 c)$. The significant repetition suppression observed in the DG/CA3 is indicative of recency signaling, and indeed the response profile is strongly consistent with this type of signal.

In line with the significant effect of familiarization and a robust interaction, we observed that, during Familiar first trials, activity was above baseline, unlike Familiar second hit trials. Indeed, a specific 

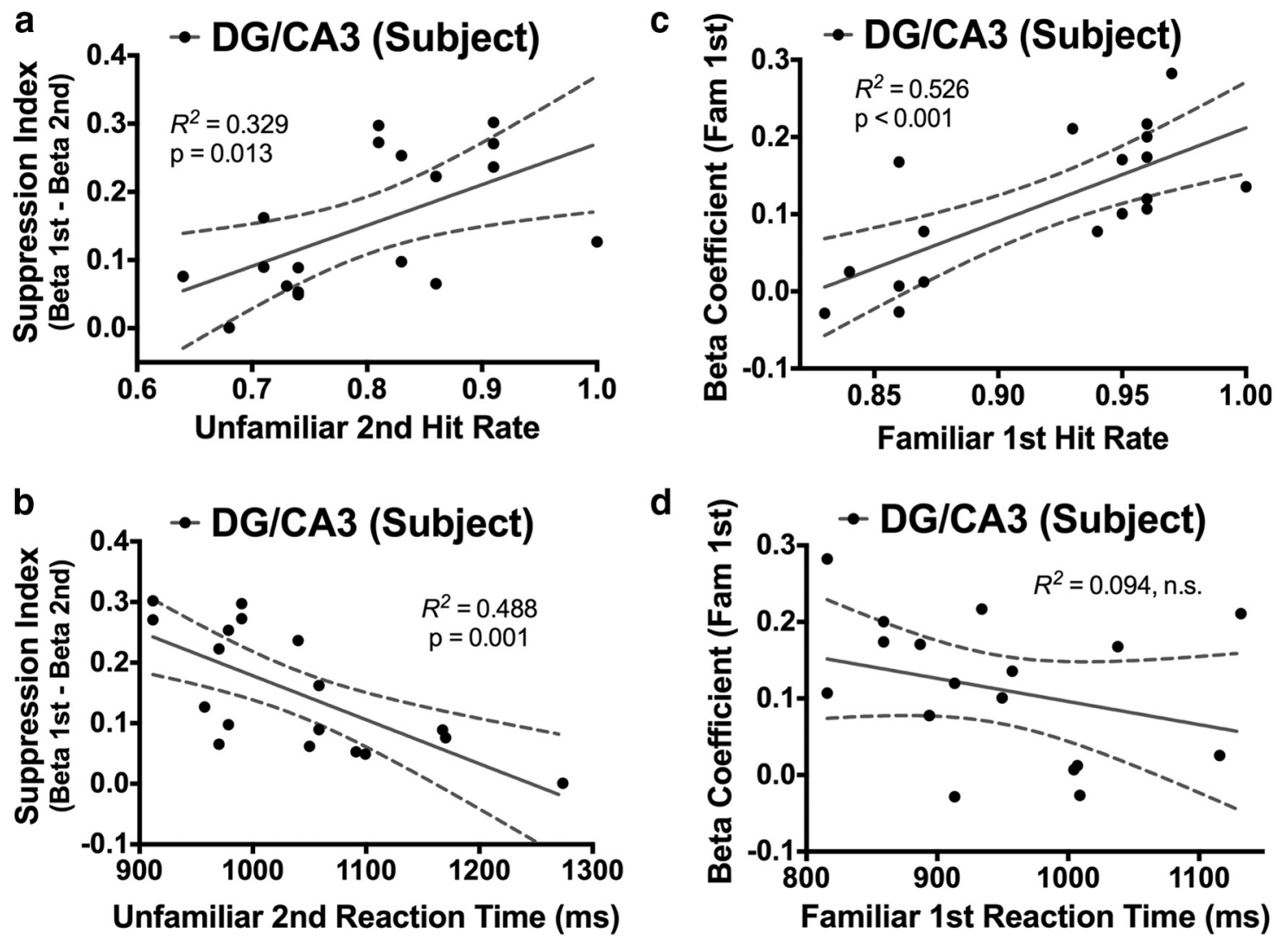

Figure 3. Correlations between the DG/CA3 and behavioral performance. During Unfamiliar second hits, the extent of repetition suppression (from the initial Unfamiliar first presentation) in the $D G / C A 3$ significantly predicted hit rate $(\boldsymbol{a})$ and reaction time $(\boldsymbol{b})$. During Familiar first hits, activity in the DG/CA3 was significantly predictive of hit rate (c) but not reaction time (d).

post hoc contrast assuming a canonical unidirectional familiarity signal was not significant under our corrected threshold $\left(F_{(1,17)}=\right.$ $8.456, p>0.05$ corrected), whereas a second contrast indicated that the positive inflection during Familiar first trials was significantly above baseline, as well as all other trials $\left(F_{(1,17)}=98.52, p<0.05\right.$ corrected).

As with CA1, we conducted an additional analysis quantifying the percentage of voxels conforming to our targeted recognition signals and confirmed that the DG/CA3 primarily exhibited recency signaling, with $43 \%$ in the left DG/CA3 and $49 \%$ in the right DG/CA3 (Fig. 2f). Comparatively, we saw very little representation of voxels conforming to familiarity or novelty signals. A large proportion of voxels significantly active during the task did not conform to our targeted signal contrasts (i.e., the other/none category), although the above analyses suggest that much of the variability in these voxels likely results from the unanticipated above-baseline Familiar first responses. This further suggests functional heterogeneity in the DG/CA3 during recognition memory.

\section{Activity in the DG/CA3 is predictive of recognition} memory performance

We next assessed the relationship between activity in the hippocampal DG/CA3 and performance on the recognition task. We first examined the data in terms of recency signals. We limited our analyses to Unfamiliar second hits (mean \pm SD accuracy, $80 \pm 10 \%$ ) because Familiar second hit performance was very close to ceiling and showed very little variability (mean \pm SD accuracy, $99 \pm 1 \%$ ). We hypothesized that the extent of repetition suppression would be predictive of Unfamiliar second hit rate. To quantify suppression of activity, we subtracted parameter estimates during Unfamiliar sec- ond hits from those during Unfamiliar first correct rejections for each subject, yielding a "suppression index" for unfamiliar trials. We found that the magnitude of repetition suppression in the DG/CA3 was positively predictive of Unfamiliar second hit rate $\left(R^{2}=0.329\right.$, $p=0.013$; Fig. $3 a)$ and negatively predictive of Unfamiliar second hit response latency $\left(R^{2}=0.488, p=0.001\right.$; Fig. $\left.3 b\right)$. That is, greater repetition suppression was strongly linked to higher hit rates and faster accurate responses.

We next assessed the relationship between the above-baseline activity during Familiar first trials and Familiar first hit rate. We found that activity in the DG/CA3 during these trials significantly predicted Familiar first hit rate $\left(R^{2}=0.526, p<0.001\right.$; Fig. $\left.3 c\right)$. However, in the case of Familiar first trials, we did not find a significant relationship between DG/CA3 activity and response latency $\left(R^{2}=0.094, p=0.215\right.$; Fig. $\left.3 d\right)$. Nonetheless, together with the repetition suppression data above, we demonstrate novel evidence that discrete BOLD signals in the human DG/CA3 are relevant to different facets of memory performance in a behavioral task. Furthermore, strikingly different signal profiles within this same hippocampal subfield are correlated with behavior on different types of recognition trials.

\section{Anterior versus posterior DG/CA3}

DG/CA3 functional heterogeneity is parsed by an anterior/posterior division

Given the heterogeneity of the two signals we demonstrated in the DG/CA3 in previous analysis, we sought to determine whether there was a spatial topography to these signals. Recall that we observed robust repetition suppression in this region, consistent with recency signaling and previous observations of activity in 

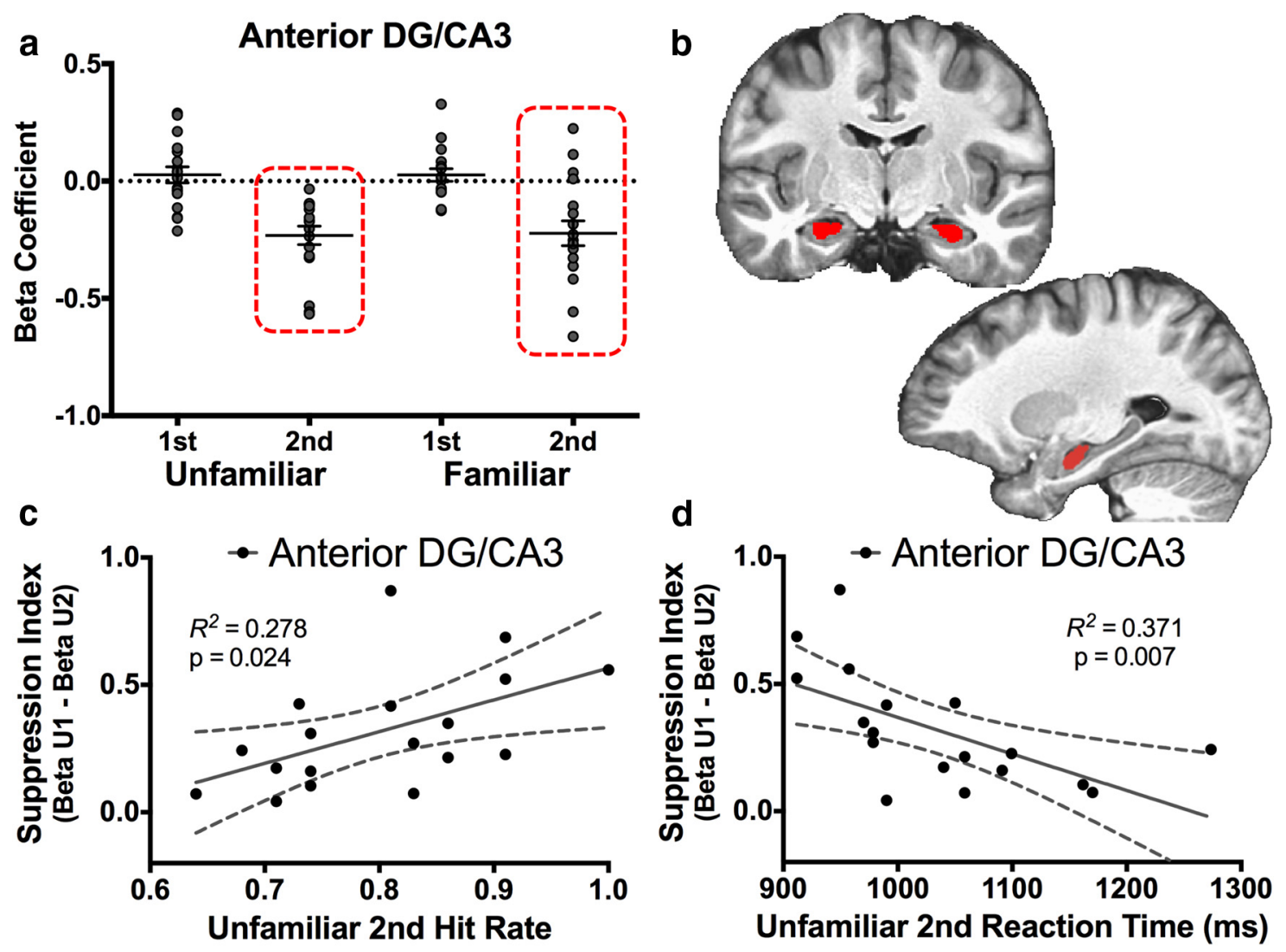

Figure 4. Recognition signals in the aDG/CA3 and correlations with behavior. $\boldsymbol{a}$, Signals in the aDG/CA3 were consistent only with recency signals. $\boldsymbol{b}$, Segmentation is displayed in the coronal and sagittal planes. Segmentation of the DG/CA3 occurred in the same manner as the whole-axis analysis in Figure 2, with the addition of the anterior/posterior division at the apex of the uncus. As with the whole-DG/CA3 analysis, repetition suppression in the aDG/CA3 was significantly predictive of both Unfamiliar second hit rate (c) and reaction time (d).

this hippocampal subfield (Bakker et al., 2008; Lacy et al., 2011). However, we also observed a curious above-baseline modulation during the first presentation of prefamiliarized items. Toward this end, we divided the DG/CA3 into anterior (aDG/CA3; Fig. $4 b$ ) and posterior (pDG/CA3; Fig. $5 c$ ) portions using the apex of the uncus as an anatomical landmark for division. This method of segmentation has been used by several previous studies in humans, and various functional distinctions between anterior and posterior hippocampus have been reported (Poppenk et al., 2013). We found strikingly dissociated signal profiles in the aDG/ $\mathrm{CA} 3$ and pDG/CA3. These profiles were consistent across hemispheres and were thus collapsed for additional analysis.

A repeated-measures ANOVA revealed that activity in the aDG/CA3 was modulated robustly by repetition $\left(F_{(1,17)}=\right.$ 47.216, $p<0.001)$. However, we did not observe a significant effect of familiarization $\left(F_{(1,17)}=0.009, p=0.927\right)$ or an interaction $\left(F_{(1,17)}=0.020, p=0.882\right)$. Thus, the aDG/CA3 demonstrated a response profile of suppression only in response to repeated items, which is highly consistent with a "pure" recency signal (Fig. 4a). We next examined the behavioral relevance of this repetition suppression from the first to second presentation, as per our whole-DG/CA3 analysis discussed previously. We found that the magnitude of repetition suppression was positively predictive of Unfamiliar second hit rate $\left(R^{2}=0.278, p=\right.$ 0.024; Fig. $4 c$ ) and negatively predictive of Unfamiliar second hit response latency $\left(R^{2}=0.371, p=0.007\right.$; Fig. $\left.4 d\right)$. That is, the repetition suppression (i.e., recency signaling) observed in the DG/CA3 and the behavioral relevance of this signal appears to have been driven by the anterior rather than the posterior portion.
A repeated-measures ANOVA revealed that activity in the pDG/CA3 was significantly modulated by both familiarization $\left(F_{(1,17)}=5.474, p=0.032\right)$ and repetition $\left(F_{(1,17)}=19.245, p<\right.$ $0.001)$. An interaction was marginal but not significant $\left(F_{(1,17)}=\right.$ 3.906, $p=0.065$ ). We observed that only the Familiar first hits condition deviated from baseline in this region and that this response was above baseline, contrary to the aDG/CA3 (Fig. 5a). Indeed, pairwise contrasts revealed that activity during Familiar first hits was significantly greater than during Familiar second hits, Unfamiliar first correct rejections, and Unfamiliar second hits (all $F>$ critical $F_{\text {Scheffé's }}$ of 13.353 , all $p<0.05$ corrected). That is, the above-baseline response observed in the DG/CA3 during Familiar first trials appears to have been driven by the posterior rather than the anterior portion.

\section{The RSC is modulated by familiarization, similar to} the $p D G / C A 3$

Although repetition suppression has been observed in the past, the above-baseline familiarity signal observed in the pDG/CA3 was indeed surprising. Because none of our MTL ROIs showed this signal profile, our next step was to pose the question of whether this signal may be shared with other regions interconnected with the MTL network. The RSC, the most posterior section of cingulate cortex, has been implicated previously in declarative memory processes, namely recollection (Johnson and Rugg, 2007; Johnson et al., 2009; Vilberg and Rugg, 2012; Fig. 5d). It is also hypothesized to be part of a wider "recollection network" together with the PHC and hippocampus (Ranganath and Ritchey, 2012). We created an anatomical mask of the RSC based on structural scans and collapsed all voxels in this region to sub- 

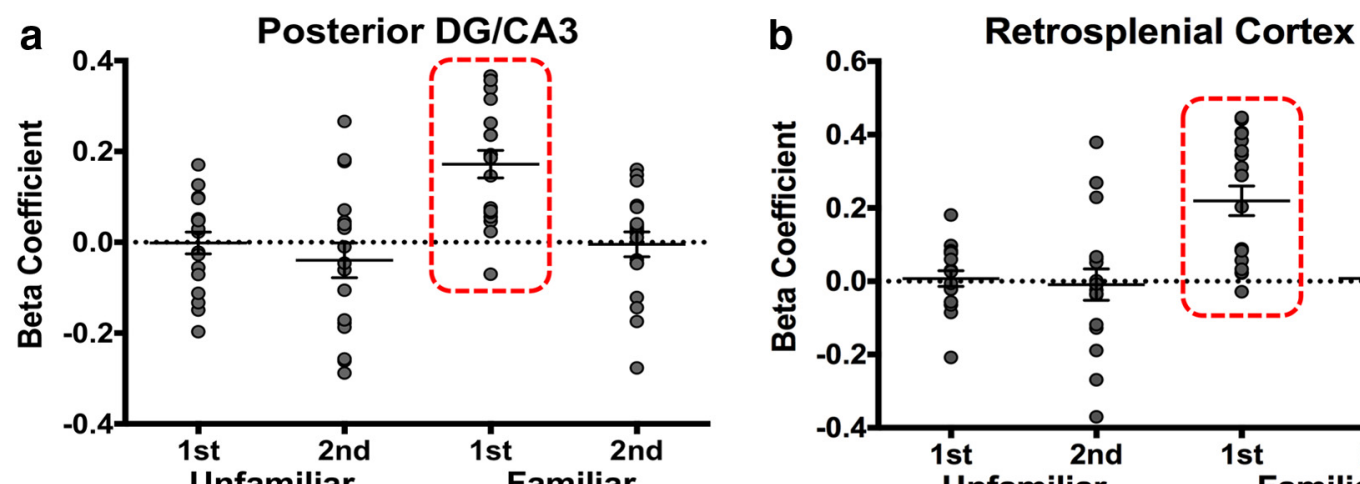

C
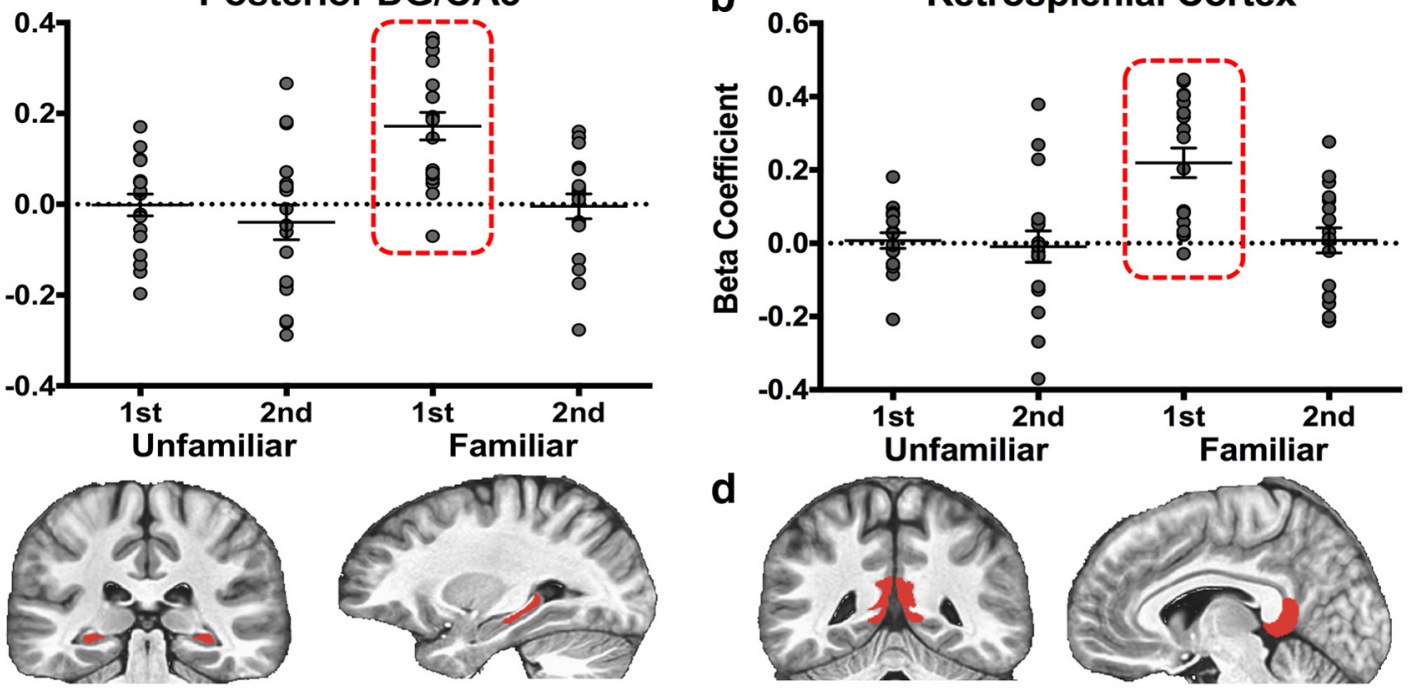

d Unfamiliar

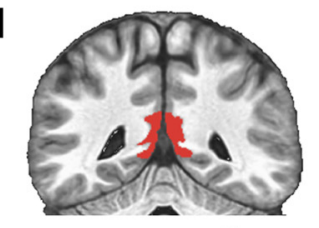

Familiar

e
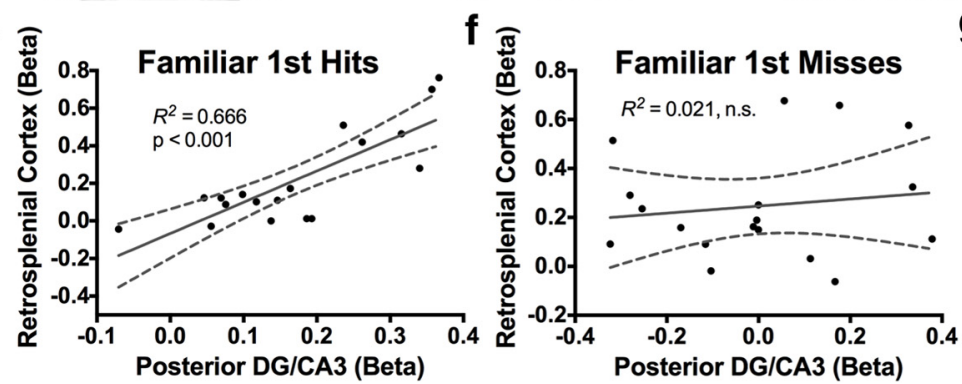

g

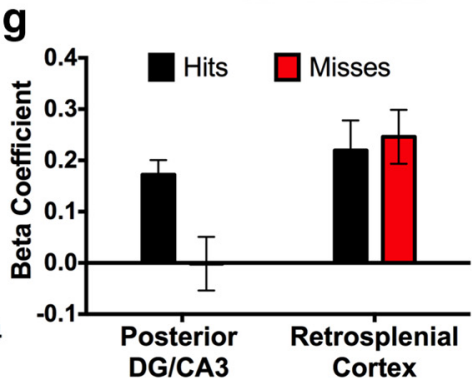

Figure 5. Recognition signals in the $\mathrm{pDG} / \mathrm{CA} 3$ and RSC and correlations with behavior. $\boldsymbol{a}$, Signals in the $\mathrm{pDG} / \mathrm{CA} 3$ were not consistent with any of the three canonical recognition signals we initially measured but were instead entirely driven by Familiar first trials. $\boldsymbol{b}$, Signals in the RSC showed a highly comparable modulation with signals in the DG/CA3 during Familiar first trials. $\boldsymbol{c}$, Segmentations of the PDG/CA3 and RSC are displayed in the coronal and sagittal planes. $\boldsymbol{d}$, Segmentation of the DG/CA3 occurred in the same manner as the whole-axis analysis in Figure 2, with the addition of the anterior/posterior division at the apex of the uncus. Activity in the pDG/CA3 and RSC were robustly correlated during Familiar first hits (e) but not Familiar first misses $(\boldsymbol{f})$. $\boldsymbol{g}$, The pDG/CA3 is modulated above baseline only during Familiar first hits but not misses. Conversely, the RSC is modulated regardless of behavioral output.

ject to exploratory analysis using our predefined recognition signal profiles. We found evidence for a signal profile in the RSC that was nearly identical to pDG/CA3 (Fig. 6b). A repeated-measures ANOVA in the RSC revealed a significant effect of familiarization $\left(F_{(1,17)}=10.749, p=0.004\right)$ and repetition $\left(F_{(1,17)}=4.596, p=\right.$ $0.047)$, as well as a significant interaction $\left(F_{(1,17)}=8.031, p=\right.$ $0.011)$. Similar to the $\mathrm{pDG} / \mathrm{CA} 3$, pairwise contrasts revealed that activity during Familiar first hits was significantly greater than during Familiar second hits, Unfamiliar first correct rejections, and Unfamiliar second hits (all $F>$ critical $F_{\text {Scheffe's }}$ of 13.353, all $p<0.05$ corrected). This suggests that the signal profile observed in the $\mathrm{pDG} / \mathrm{CA} 3$ was not spurious by providing evidence of another region demonstrating highly comparable patterns of activity.

The RSC and pDG/CA3 may work together in support of recognition performance

Given the strikingly similar response profiles between the pDG/ CA3 and RSC, we tested correlations between these regions during Familiar first recognition trials. We observed a significant correlation between the pDG/CA3 and RSC during Familiar first hits $\left(R^{2}=0.666, p<0.001\right.$; Fig. $\left.5 e\right)$, but this correlation was absent during Familiar first misses $\left(R^{2}=0.021, p=0.569\right.$; Fig. $5 f)$. This difference between correlations during Familiar first hits and Familiar first misses was significant $(Z=2.74, p=$ $0.031)$. Thus, the $\mathrm{pDG} / \mathrm{CA} 3$ and RSC may share a related computation during Familiar first trials but only during successful recognition. Additionally, the RSC shows an above-baseline response during Familiar first trials regardless of whether the response was a hit or miss. However, the pDG/CA3 showed above-baseline activity only during hits, which differed significantly from misses $\left(t_{(17)}=3.105, p=0.003\right.$; Fig. $\left.5 g\right)$. That is, the RSC was modulated by Familiar first trials regardless of behavioral performance, whereas modulations of activity in the pDG/ CA3 were contingent on successful recognition.

Like in the aDG/CA3, we sought to characterize the possible behavioral relevance of these signals in the pDG/CA3. We examined activity during Familiar first hits only and observed that the pDG/CA3 was predictive of Familiar first hit rate $\left(R^{2}=0.642\right.$, $p<0.001$; Fig. $6 a)$ and response latency $\left(R^{2}=0.526, p<0.001\right.$; Fig. $6 b)$. In contrast, the RSC did not significantly correlate with either Familiar first hit rate $\left(R^{2}=0.011, p=0.663\right.$; Fig. $\left.6 c\right)$ or response latency $\left(R^{2}<0.001, p=0.980\right.$; Fig. $\left.6 d\right)$. Together with the dissociation between Familiar first hits and Familiar first misses, these correlations suggest that the pDG/CA3, but not the RSC, was predictive of behavior during recognition of familiarized items when viewed for the first time during the task.

\section{MTL cortical ROIs}

The PRC shows familiarity and novelty signals

Segmentation of MTL cortices can be seen in a representative anterior (Fig. 7a) and posterior (Fig. 7b) slice. A repeatedmeasures ANOVA in the left PRC revealed significant effects of familiarization $\left(F_{(1,17)}=21.048, p<0.001, \eta_{p}^{2}=0.553\right)$ and repetition $\left(F_{(1,17)}=10.435, p=0.005, \eta_{p}^{2}=0.380\right)$. An interac- 

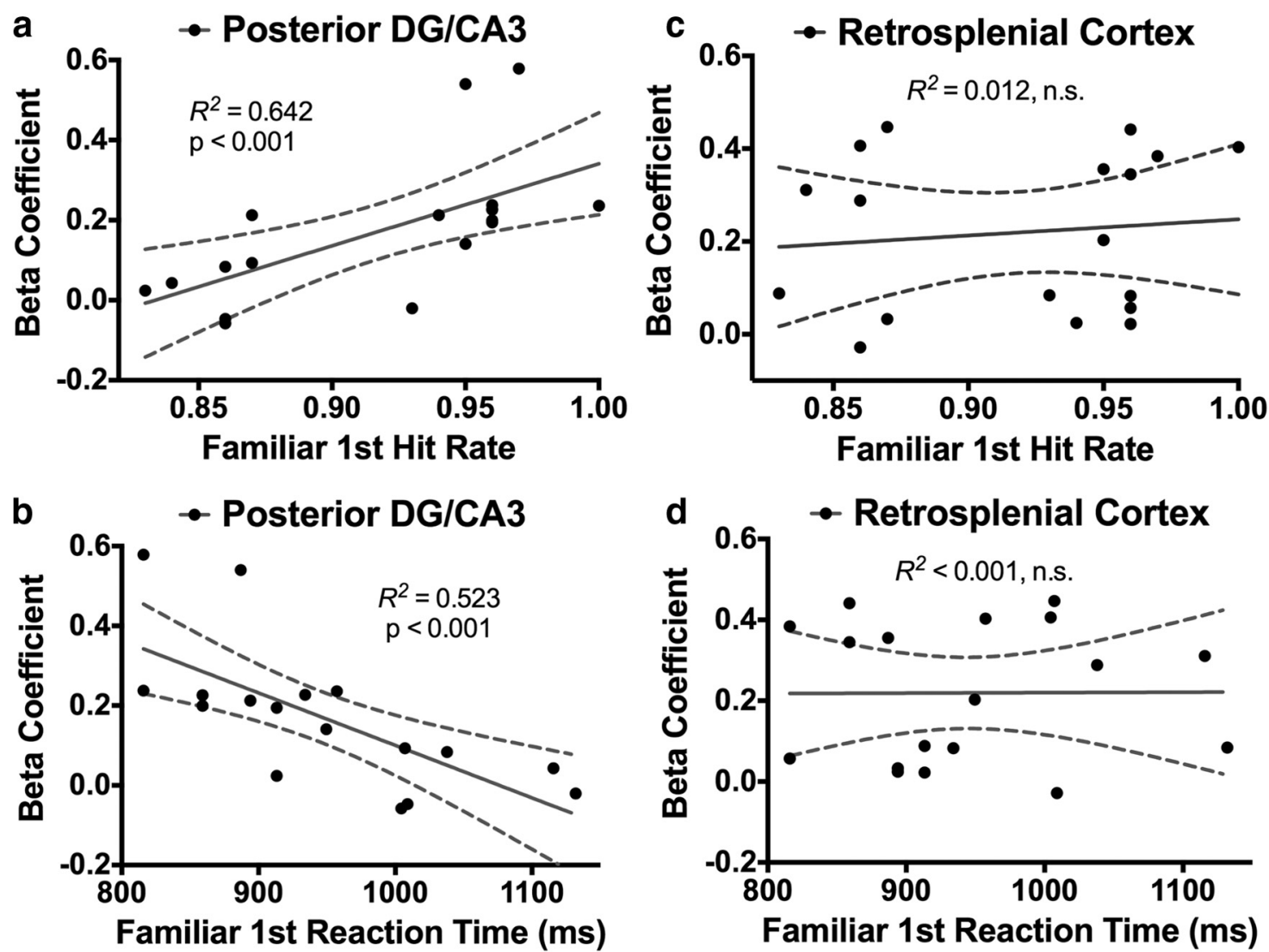

Figure 6. Behavioral correlates of activity in the $\mathrm{pDG} / \mathrm{CA} 3$ and RSC. Activity in the $\mathrm{DDG} / \mathrm{CA} 3$ was significantly predictive of Familiar first hit rate $(\boldsymbol{a})$ and reaction time (b). In contrast, the RSC was predictive of neither Familiar first hit rate (c) nor reaction time $(\boldsymbol{d})$.

tion was marginal but not significant $\left(F_{(1,17)}=3.256, p=0.089\right.$, $\eta_{p}^{2}=0.161$; Fig. $\left.7 c\right)$. Observing the data in Figure $7 c$, it appears that the most robust suppression occurred in response to familiarized stimuli (Familiar first and Familiar second hit trials), with some modulation occurring in response to Unfamiliar second hit trials. A post hoc contrast revealed that activity in the left PRC was significantly consistent with novelty signaling $\left(F_{(1,17)}=56.654\right.$, $p<0.05$ corrected). Moreover, a directed contrast assessing recency here was not significant given our corrected threshold $\left(F_{(1,17)}=11.624, p>0.05\right.$ corrected $)$, suggesting that the significant main effect of repetition was driven mostly by general novelty responses.

Consistent with this account, we found that the majority of voxels in the left PRC responded most robustly in accordance with familiarity signals at $44 \%$, and $21 \%$ of voxels most robustly demonstrated novelty signaling (Fig. $7 f$ ). In contrast, very few voxels were significantly designated as demonstrating recency signals.

Unlike the left PRC, a repeated-measures ANOVA in the right PRC revealed a significant effect of familiarization $\left(F_{(1,17)}=\right.$ $\left.16.658, p=0.001, \eta_{p}^{2}=0.495\right)$. However, we observed no effect of repetition $\left(F_{(1,17)}=0.165, p=0.689, \eta_{p}^{2}=0.011\right)$ and no interaction $\left(F_{(1,17)}=0.035, p=0.854, \eta_{p}^{2}=0.002\right.$; Fig. $\left.4 c\right)$. Furthermore, we did not observe a significant novelty response profile via a post hoc contrast $\left(F_{(1,17)}=3.502, p>0.05\right.$ corrected $)$. Additionally, of all voxels significantly active during the task, $66 \%$ most robustly demonstrated with familiarity signals, whereas very few were consistent with recency or novelty signaling (Fig. $7 f$ ). These marked differences between the left and right PRC are likely to have driven the effect of hemisphere and the interaction between region and hemisphere in our first-level ANOVA. Together, these data suggest that the PRC is overall strongly modulated by familiarity, consistent with previous studies (Brown and Bashir, 2002; Diana et al., 2007; Haskins et al., 2008). However, as evinced by our more heterogeneous left PRC ROI, this region may support other forms of recognition memory, such as novelty signaling.

The PHC shows mixed recognition signals

Activity in the PHC was highly consistent across hemispheres and was collapsed for the following analyses. A repeated-measures ANOVA revealed significant effects of familiarization $\left(F_{(1,17)}=\right.$ 9.355, $\left.p=0.007, \eta_{p}^{2}=0.355\right)$ and repetition $\left(F_{(1,17)}=16.443, p=\right.$ $\left.0.001, \eta_{p}^{2}=0.492\right)$. We did not observe a significant interaction $\left(F_{(1,17)}=0.175, p=0.681, \eta_{p}^{2}=0.010\right.$; Fig. $\left.7 d\right)$. Post hoc contrasts revealed a response profile significantly consistent with novelty signaling $\left(F_{(1,17)}=32.265, p<0.05\right.$ corrected $)$ and confirmed the presence of specific recency $\left(F_{(1,17)}=23.317, p<0.05\right.$ corrected $)$ and familiarization $\left(F_{(1,17)}=21.092, p<0.05\right.$ corrected $)$ response profiles. Thus, the bilateral PHC appeared to express a heterogeneous mixture of recognition signals in this experiment.

Consistent with this analysis, we found a fairly even mixture of voxels across the left and right PHC that conformed to our three targeted recognition signals (Fig. $7 \mathrm{~g}$ ). Most voxels were responsive to recency (left PHC, 24\%; right PHC, 33\%) and novelty (left $\mathrm{PHC}, 30 \%$; right $\mathrm{PHC}, 23 \%$ ) signals, although a fair proportion were responsive mainly to familiarization (left $\mathrm{PHC}, 17 \%$; right PHC, 21\%). We note that, among all ROIs, the bilateral PHC was the only region in which voxel overlap via this designation exceeded $20 \%$. We take this as additional support of a great deal of 

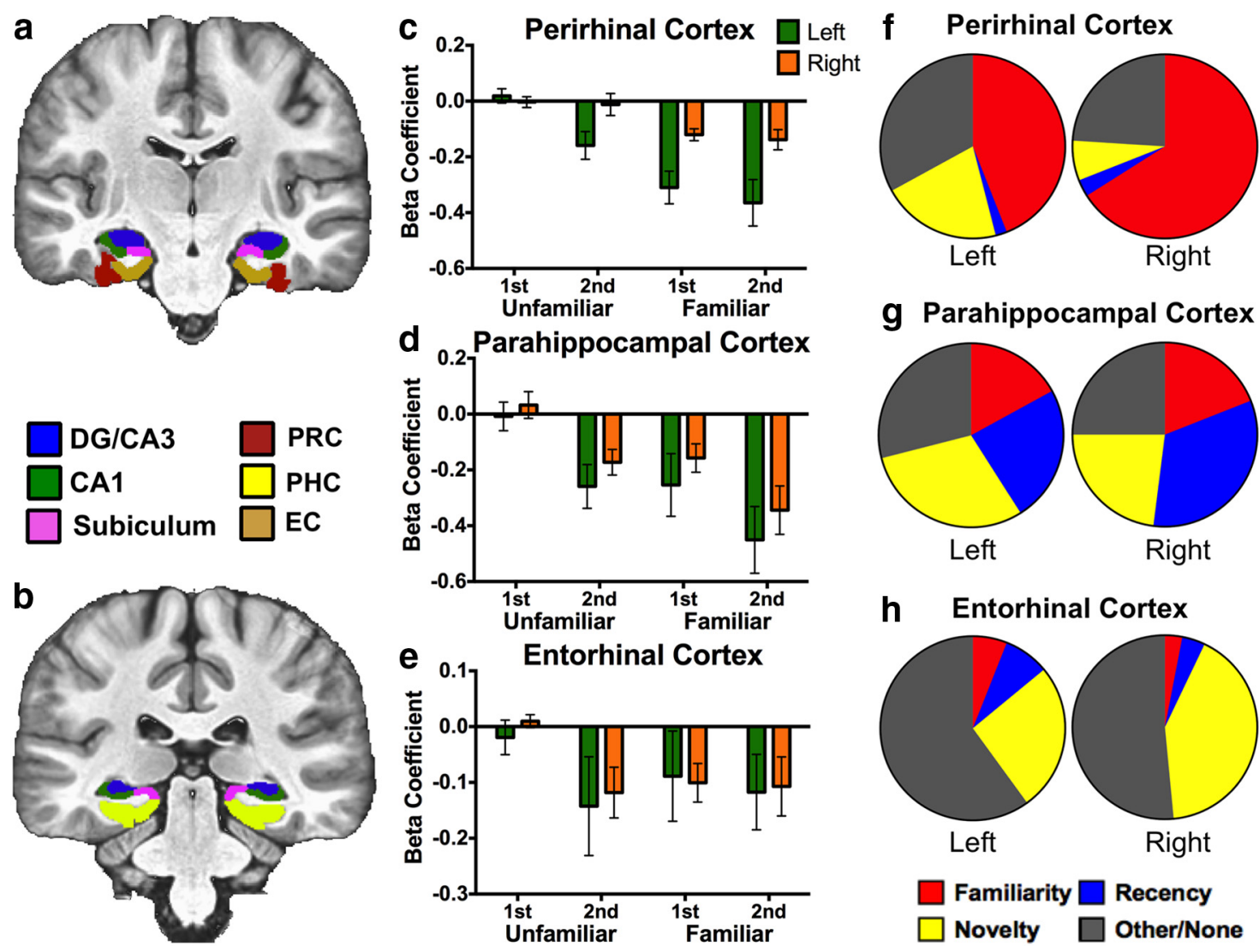

Figure 7. Recognition signals in MTL cortices. Segmentation is displayed in two representative anterior (a) and posterior (b) slices. Activity during Novel Foil trials was used as baseline (zero). c, Activity in the left PRC was consistent with both familiarity and match/mismatch signaling, whereas activity in the right PRC was only consistent with familiarity signaling. $\boldsymbol{d}$, Activity in the bilateral PHC was consistent with all recognition signals examined, suggesting a mixture of familiarity, recency, and match/mismatch signals. $\boldsymbol{e}$, Activity in the bilateral EC was consistent with match/ mismatch signaling. $\boldsymbol{f}-\boldsymbol{h}$, Percentage of voxels conforming to each of the three canonical recognition signal profiles or other/none across MTL cortices at a familywise threshold of $p=0.05$. Voxels were designated based on whichever contrast yielded the largest significant $F$ statistic.

functional heterogeneity in this region of the MTL cortex during recognition memory.

The EC demonstrates novelty signaling

As with previous analyses, we collapsed the EC across hemispheres because of highly comparable response profiles. A repeated-measures ANOVA revealed a significant effect of repetition $\left(F_{(1,17)}=4.934, p=0.041, \eta_{p}^{2}=0.225\right)$ but no effect of familiarization $\left(F_{(1,17)}=1.189, p=0.291, \eta_{p}^{2}=0.177\right.$; Fig. $\left.7 e\right)$. We observed a marginal but nonsignificant interaction $\left(F_{(1,17)}=\right.$ $\left.4.224, p=0.056, \eta_{p}^{2}=0.199\right)$. Specific post hoc contrasts revealed significant novelty signaling $\left(F_{(1,17)}=14.021, p<0.05\right.$ corrected), whereas activity here was not consistent with recency or familiarity (both $p>0.05$ corrected).

Assessing the relative proportions of recognition signal profiles across the EC, we indeed found a large subset of voxels to show activity consistent with novelty signaling (left EC, 26\%; right EC, 44\%) and very few voxels consistent with familiarity or recency signaling (Fig. $7 \mathrm{~h}$ ). We observed a large proportion of noncategorized voxels within this region, which can to some extent be attributed to a lower signal-to-noise ratio and greater signal dropout in the EC (note the relatively large variance in this region via Fig. 7e).

\section{Discussion}

In this experiment, we varied stimulus familiarization and repetition in a task designed to tax multiple facets of recognition memory. Our aim was to characterize recognition-related signals in hippocampal subfields and to further characterize signals in MTL cortices using high-resolution fMRI. Behavioral results indicated a general benefit conferred by familiarization with a stimulus set and a benefit of stimulus repetition. This was evident in an increase in hit rate and a decrease in reaction time in response to familiarized stimuli, and the same basic effects were observed as a function of repetition. These data demonstrate, rather unsurprisingly, that pretest encounters with a stimulus and within-test repetition enhance one's ability to recognize that stimulus (but see Reagh and Yassa, 2014 for a demonstration of possible pitfalls of repetition).

fMRI analyses revealed various recognition-related signals within our hippocampal and MTL cortical ROIs. We observed novelty signals in the left CA1. This is consistent with match/ mismatch detection in this region, in agreement with previous reports (Duncan et al., 2012). However, as noted above in Results, we opt to describe the signaling as novelty rather than match/mismatch detection in light of aforementioned distinctions and because the latter implies specific computational interplay between CA3 and CA1 that our fMRI data do not address (Lisman, 1999). We did not observe any significant modulations in either the left or right subiculum. Also consistent with previous studies, we found that the PRC robustly signaled familiarity (Brown and Bashir, 2002; Diana et al., 2007; Haskins et al., 2008; Staresina et al., 2011). We also observed novelty signals in the EC and a heterogeneous mixture of signal profiles in the PHC.

Furthermore, we characterized the relative proportions of voxels conforming to different signals across our ROIs. This con- 
tributes to our understanding of MTL cortices in support of recognition memory by evaluating (insofar as BOLD fMRI is able) the extent to which given regions express certain signal profiles. In the present data, we did not conduct specific analyses over areas of overlap, and, as mentioned in Results, only the PHC featured particularly high degrees of overlap among signal profile contrasts. We feel that this approach is informative, and we chose our winner-take-all approach to voxel designation for the sake of a more straightforward and tractable discussion of these data. We acknowledge that this approach intentionally overlooks possible fine-grained nuances in voxel overlap.

Our most intriguing observation was that of functional heterogeneity in the DG/CA3. We observed robust recency signals in this hippocampal subfield. This response profile is somewhat consistent with the hypothesized role of the DG/CA3 in pattern separation (Yassa and Stark, 2011), and repetition suppression in this region to repeated information (but not highly similar lure images) has been used to index this computation (Bakker et al., 2008; Lacy et al., 2011; LaRocque et al., 2013). Furthermore, we demonstrate novel evidence for a relationship between the extent of repetition suppression in the DG/CA3 and recognition performance. It is unclear why this suppression was not observed in response to familiarized images presented for a first time within a session. Surprisingly, during these trials, we observed a categorically different response profile in the DG/CA3 in which activity was modulated robustly above baseline. Similar to the recency signaling, this above-baseline modulation was predictive of recognition performance on its respective trial type (Familiar first). Most conservatively, we can say that these results demonstrate that the human DG/CA3 is functionally heterogeneous.

Although not our aim at the outset of this study, additional analyses revealed a striking dissociation between the anterior and posterior portions of the DG/CA3 subregion. aDG/CA3 robustly signaled recency such that repetition of stimuli within session (regardless of general familiarity) elicited suppression relative to baseline. As with the full DG/CA3 data, the extent of this suppression was highly predictive of behavioral accuracy and response latency during the subsequent presentation of an unfamiliar image (as noted in Results, familiarized images were beyond such analysis because of ceiling performance). In contrast with the aDG/CA3, the pDG/CA3 demonstrated activity that was not consistent with any of the three canonical recognition signals we have described. Quite unexpectedly, and unlike the aDG/CA3, this subregion was responsive only to familiarized items seen for a first time within session. Also unlike its anterior counterpart, the pDG/CA3 only demonstrated above-baseline responses. To our knowledge, this is the first evidence of such a stark contrast between anterior and posterior portions of the DG/CA3 subfield (or any subfield) of the human hippocampus, particularly in terms of directionality of the signal. Particularly, we observed repetition suppression only in the aDG/CA3 but a modulation in $\mathrm{pDG} / \mathrm{CA} 3$ in the opposite direction.

What mechanisms or computations might underlie this difference? Long-axis specialization of the hippocampus has received a fair amount of recent attention. A thorough and in-depth review by Poppenk et al. (2013) discussed various dissociations and explanations for those dissociations, such as the amount of emotional or spatial content being processed (for details on the wide range of findings, see their review). Poppenk et al. (2013) propose a synthesis model based on the scale of information being processed, with the anterior hippocampus operating over coarse, schematic informa- tion and the posterior hippocampus operating over elaboration of details. This model eloquently accounts for many reported dissociations on the basis of different structural connectivity and functional properties along the hippocampal axis.

Our data are actually quite consistent with their account, and given its mechanistic grounding, we favor an explanation along these lines. Specifically, repetition suppression in the aDG/CA3 could be considered as a coarse comparison with recently encountered information (i.e., information being actively maintained, "online" in the MTL memory network within session). This coarse sort of representation might be all that is necessary to drive the experience of recognition in situations in which encountered information features high overlap with something already online. In contrast, signals in the pDG/CA3 specific to familiarized items after a $24 \mathrm{~h}$ delay may be indicative of a more "constructive" process of piecing together information in memory and comparing that with a current stimulus. This very different type of memory signal may drive recognition in this different, more actively "reconstructive" sense. Perhaps for the Familiar second trials, this elaborative reconstructive process is no longer necessary because the representation is now online. We note that this remains speculation with respect to the data at hand. Future studies more targeted at such a long-axis dissociation can further elucidate the consistency of DG/CA3 activity with the model proposed by Poppenk et al. (2013).

There are other potential explanations for this distinction. Perhaps the experience of recalling information over a $24 \mathrm{~h}$ delay taxes recollection to a greater extent than within-session recognition, which may tax familiarity-based memory judgments to a greater extent, in the sense of the dual process model. However, given differences between our familiar stimuli and the dual process sense of "familiarity," we are hesitant to make such a link (however, see the discussion below relating to the RSC on this matter). Another possibility is that the amount of time passed between the study and the test itself drives different recognition signal profiles in the hippocampus and along its axis, although virtually no existing studies have parametrically tested such a mechanism in a controlled experiment.

Interestingly and unexpectedly, the RSC also showed a positive inflection only during familiarized first presentation trials, which was highly comparable with the pDG/CA3 signal. Indeed, activity in the pDG/CA3 and RSC appeared to be highly correlated, but this correlation existed only during target hits and not during misses. We attribute this to the fact that the RSC responded identically regardless of judgment accuracy, whereas this above-baseline inflection in the pDG/CA3 was dependent on successful recognition. Consistent with this behavioral conditionality, we observed that the $\mathrm{pDG} / \mathrm{CA} 3$ was predictive of recognition of these familiarized items presented a first time within session, but the RSC was not. An additional novel contribution of this study is the identification of related but dissociable computations in the DG/CA3 and RSC.

Naturally, this raises an important question: what computation could the pDG/CA3 and RSC be sharing? Previous studies involving the RSC may offer some perspective. This cortical region has primarily been implicated in recollection (Yonelinas et al., 2005; Daselaar et al., 2006; Diana et al., 2007; Johnson et al., 2009) and is included in a larger putative recollection network of brain regions distinct from those that support familiarity-based memory judgments (Ranganath and Ritchey, 2012). This being the case, the involvement of the RSC may implicate a recollection-like process as having occurred. This may offer clues as to the functional role of the pDG/CA3, at least in the context of 
this paradigm. That is, perhaps the RSC is involved in a process that would support recollection of studied items studied $24 \mathrm{~h}$ previously and perhaps a reconstruction of the relevant neural representations. If this is the case, then perhaps the pDG/CA3 is also involved in such a process, although the output of the DG/ CA3 rather than the RSC seemed to drive behavior. Although data on connectivity between the RSC and hippocampal subfields is limited, there do appear to be reciprocal connections between the RSC and the hippocampus, as well as cortices along the parahippocampal gyrus (Wyss and Van Groen, 1992; Kobayashi and Amaral, 2003, 2007). The most prominent connections between the RSC and MTL regions appear to be mediated through the subiculum and EC (Aggleton et al., 2012). Ultimately, the nature and extent of the computational interactions between the pDG/ CA3 and RSC observed here will be a matter of future research targeted at interplay between the RSC and the MTL.

In conclusion, this study used high-resolution fMRI to observe and quantify various recognition-related signals throughout human hippocampal subfields and MTL cortices. Furthermore, we linked activity in the hippocampal DG/CA3 to behavior and found dissociable recognition-related signals in the hippocampal DG/CA3 subfield along the hippocampal longitudinal axis. We also observed robust and dissociable relationships between the $\mathrm{aDG} / \mathrm{CA} 3$ and $\mathrm{pDG} / \mathrm{CA} 3$ and respective behavioral indices of recognition. Future studies will be needed to further elucidate the specialized roles of hippocampal subregions along its longitudinal axis. In particular, this study raises questions about the nature of dissociations within the DG/CA3 along the longitudinal axis that may be attributable to the processes required to retrieve the information (i.e., the extent to which elements of a memory trace need to be "reconstructed"), greater reliance on recollection versus familiarity, or the passage of time itself. Experiments can be designed to explicitly tease apart these possibilities.

\section{References}

Aggleton JP, Wright NF, Vann SD, Saunders RC (2012) Medial temporal lobe projections to the retrosplenial cortex of the macaque monkey. Hippocampus 22:1883-1900. CrossRef Medline

Avants BB, Tustison N, Song G (2009) Advanced normalization tools (ANTS). Philadelphia: Penn Image Computing and Science Laboratory; University of Pennsylvania.

Bakker A, Kirwan CB, Miller M, Stark CEL (2008) Pattern separation in the human hippocampal CA3 and dentate gyrus. Science 319:1640-1642. CrossRef Medline

Barker GRI, Warburton EC (2011) When is the hippocampus involved in recognition memory? J Neurosci 31:10721-10731. CrossRef Medline

Brainard DH (1997) The Psychophysics Toolbox. Spat Vis 10:433-436. Medline

Brown MW, Aggleton JP (2001) Recognition memory: what are the roles of the perirhinal cortex and hippocampus? Nat Rev Neurosci 2:51-61. CrossRef Medline

Brown MW, Bashir ZI (2002) Evidence concerning how neurons of the perirhinal cortex may affect familiarity discrimination. Philos Trans R Soc Lond B Biol Sci 357:1083-1095. CrossRef Medline

Buffalo EA, Reber PJ, Squire LR (1998) The human perirhinal cortex and recognition memory. Hippocampus 8:330-339. CrossRef Medline

Clark RE, Zola SM, Squire LR (2000) Impaired recognition memory in rats after damage to the hippocampus. J Neurosci 20:8853-8860. Medline

Cox RW (1996) AFNI: software for analysis and visualization of functional magnetic resonance neuroimages. Comput Biomed Res 29:162-173. CrossRef Medline

Daselaar SM, Fleck MS, Cabeza R (2006) Triple dissociation in the medial temporal lobes: recollection, familiarity, and novelty. J Neurophysiol 96: 1902-1911. CrossRef Medline

Diana RA, Yonelinas AP, Ranganath C (2007) Imaging recollection and familiarity in the medial temporal lobe: a three-component model. Trends Cogn Sci 11:379-386. CrossRef Medline
Diana RA, Yonelinas AP, Ranganath C (2012) Adaptation to cognitive context and item information in the medial temporal lobes. Neuropsychologia 50:3062-3069. CrossRef Medline

Duncan K, Curtis C, Davachi L (2009) Distinct memory signatures in the hippocampus: intentional states distinguish match and mismatch enhancement signals. J Neurosci 29:131-139. CrossRef Medline

Duncan K, Ketz N, Inati SJ, Davachi L (2012) Evidence for area CA1 as a match/mismatch detector: a high-resolution fMRI study of the human hippocampus. Hippocampus 22:389-398. CrossRef Medline

Duvernoy H (1998) The human hippocampus: functional anatomy, vascularization, and serial sections with MRI. Berlin: Springer.

Düzel E, Vargha-Khadem F, Heinze HJ, Mishkin M (2001) Brain activity evidence for recognition without recollection after early hippocampal damage. Proc Natl Acad Sci U S A 98:8101-8106. CrossRef Medline

Eichenbaum H, Yonelinas AP, Ranganath C (2007) The medial temporal lobe and recognition memory. Annu Rev Neurosci 30:123-152. CrossRef Medline

Fortin NJ, Wright SP, Eichenbaum H (2004) Recollection-like memory retrieval in rats is dependent on the hippocampus. Nature 431:188-191. CrossRef Medline

Hannula DE, Ranganath C (2008) Medial temporal lobe activity predicts successful relational memory binding. J Neurosci 28:116-124. CrossRef Medline

Haskins AL, Yonelinas AP, Quamme JR, Ranganath C (2008) Perirhinal cortex supports encoding and familiarity-based recognition of novel associations. Neuron 59:554-560. CrossRef Medline

Johnson JD, Rugg MD (2007) Recollection and the reinstatement of encoding-related cortical activity. Cereb Cortex 17:2507-2515. CrossRef Medline

Johnson JD, McDuff SGR, Rugg MD, Norman KA (2009) Recollection, familiarity, and cortical reinstatement: a multivoxel pattern analysis. Neuron 63:697-708. CrossRef Medline

Jutras MJ, Buffalo EA (2010) Recognition memory signals in the macaque hippocampus. Proc Natl Acad Sci U S A 107:401-406. CrossRef Medline

Kobayashi Y, Amaral DG (2003) Macaque monkey retrosplenial cortex: II. Cortical afferents. J Comp Neurol 446:48-79. CrossRef Medline

Kobayashi Y, Amaral DG (2007) Macaque monkey retrosplenial cortex: III. Cortical efferents. J Comp Neurol 502:810-833. CrossRef Medline

Kumaran D, Maguire EA (2006) An unexpected sequence of events: mismatch detection in the human hippocampus. PLoS Biol 4:e424. CrossRef Medline

Kumaran D, Maguire EA (2007) Match mismatch processes underlie human hippocampal responses to associative novelty. J Neurosci 27:85178524. CrossRef Medline

Lacy JW, Yassa MA, Stark SM, Muftuler LT, Stark CE (2011) Distinct pattern separation related transfer functions in human CA3/dentate and CA1 revealed using high-resolution fMRI and variable mnemonic similarity. Learn Mem 18:15-18. CrossRef Medline

LaRocque KF, Smith ME, Carr VA, Witthoft N, Grill-Spector K, Wagner AD (2013) Global similarity and pattern separation in the human medial temporal lobe predict subsequent memory. J Neurosci 33:5466-5474. CrossRef Medline

Lisman JE (1999) Relating hippocampal circuitry to function: recall of memory sequences by reciprocal dentate-CA3 interactions. Neuron 22: 233-242. CrossRef Medline

Manns JR, Hopkins RO, Reed JM, Kitchener EG, Squire LR (2003) Recognition memory and the human hippocampus. Neuron 37:171-180. CrossRef Medline

Murray EA, Bussey TJ (1999) Perceptual-mnemonic functions of the perirhinal cortex. Trends Cogn Sci 3:142-151. CrossRef Medline

Murray EA, Baxter MG, Gaffan D (1998) Monkeys with rhinal cortex damage or neurotoxic hippocampal lesions are impaired on spatial scene learning and object reversals. Behav Neurosci 112:1291-1303. CrossRef Medline

Norman G, Eacott MJ (2005) Dissociable effects of lesions to the perirhinal cortex and the postrhinal cortex on memory for context and objects in rats. Behav Neurosci 119:557-566. CrossRef Medline

Poppenk J, Evensmoen HR, Moscovitch M, Nadel L (2013) Long-axis specialization of the human hippocampus. Trends Cogn Sci 17:230-240. CrossRef Medline

Ranganath C, Ritchey M (2012) Two cortical systems for memory-guided behaviour. Nat Rev Neurosci 13:713-726. CrossRef Medline 
Reagh ZM, Yassa MA (2014) Repetition strengthens target recognition but impairs similar lure discrimination: evidence for trace competition. Learn Mem 21:342-346. CrossRef Medline

Staresina BP, Duncan KD, Davachi L (2011) Perirhinal and parahippocampal cortices differentially contribute to later recollection of object- and scene-related event details. J Neurosci 31:8739-8747. CrossRef Medline

Suzuki WA, Zola-Morgan S, Squire LR, Amaral DG (1993) Lesions of the perirhinal and parahippocampal cortices in the monkey produce longlasting memory impairment in the visual and tactual modalities. J Neurosci 13:2430-2451. Medline

Vilberg KL, Rugg MD (2012) The neural correlates of recollection: transient versus sustained FMRI effects. J Neurosci 32:15679-15687. CrossRef Medline

Wan H, Aggleton JP, Brown MW (1999) Different contributions of the hippocampus and perirhinal cortex to recognition memory. J Neurosci 19: 1142-1148. Medline

Wyss JM, Van Groen T (1992) Connections between the retrosplenial cor- tex and the hippocampal formation in the rat: a review. Hippocampus 2:1-11. CrossRef Medline

Yassa MA, Stark CEL (2008) Multiple signals of recognition memory in the medial temporal lobe. Hippocampus 18:945-954. CrossRef Medline

Yassa MA, Stark CEL (2011) Pattern separation in the hippocampus. Trends Neurosci 34:515-525. CrossRef Medline

Yonelinas AP, Kroll NEA, Quamme JR, Lazzara MM, Sauvé MJ, Widaman KF, Knight RT (2002) Effects of extensive temporal lobe damage or mild hypoxia on recollection and familiarity. Nat Neurosci 5:1236-1241. CrossRef Medline

Yonelinas AP, Otten LJ, Shaw KN, Rugg MD (2005) Separating the brain regions involved in recollection and familiarity in recognition memory. J Neurosci 25:3002-3008. CrossRef Medline

Zola-Morgan S, Squire LR, Amaral DG, Suzuki WA (1989) Lesions of perirhinal and parahippocampal cortex that spare the amygdala and hippocampal formation produce severe memory impairment. J Neurosci 9:4355-4370. Medline 